\title{
Research on Transformation Maturity of Scientific Research Achievements in Power Industry Based on Neural Network Model
}

\author{
Yujing Gong*, Liyong Duan, Yongjun Liu \\ China Southern Power Grid Scientific Research Institute, Guangzhou, 510700, China \\ *Corresponding Author
}

Keywords: Neural network, Power industry, Scientific research, Transformation, Maturity

\begin{abstract}
The development of the economy is always accompanied by the advancement of science and technology, and it is supported by science and technology. Scientific research is changing with each passing day, and it is developing at a rapid pace. However, to truly evaluate the economic value of a scientific research achievement, its own benefits need to be measured by an evaluation system that transforms scientific research results. Facing the current situation that China's scientific research achievements are not mature enough, this paper uses neural network model to study the transformation maturity of scientific research achievements in the power industry. Through the research and analysis of this paper, more measures and policies should be implemented to accelerate the maturity of the transformation of scientific research results.
\end{abstract}

\section{Introduction}

NASA introduced the concept of technology maturity(Technology Readiness Levels,TRL) in the 1970s. The maturity of science and technology has a clearer framework.Scientific research results are a kind of knowledge-based intangible assets. The composition of such assets mainly depends on highly intensive knowledge, intelligence, technology and skills [1].Technology transfer is an important process for the economic, social and ecological benefits of the scientific research achieved [2].Accelerate the transformation of scientific research results of electric power research institutes, promote the value-added of scientific research achievements, promote the pace of power technology innovation, and promote the development of intelligent and informationized power enterprises and the realization of smart grids [3].Scientific monitoring and evaluation of the results of scientific and technological activities and their transformation and application is an important basis for the formulation of science and technology policies [4].That is to say, the true value of scientific research results is in the real application, from the scientific research field to the economic industrialization.

The transformation of scientific and technological achievements is an indispensable link in the application of scientific and technological achievements to production practice. It is an important means to realize the combination of science and technology and economic development and promote social and economic development in China [5].He Defang [6] through the comparative analysis of related concepts such as the transformation of scientific and technological achievements, technology transfer, and application of scientific and technological achievements, pointed out that the transformation of scientific and technological achievements is based on technological success, and through the process of experimental development, commercialization and industrialization, economic benefits and society are produced. It can be seen that the research results need to go through an evolutionary process from research and development to application, and some problems are often encountered in evolution.Rogers [7] proposed the Theory of Diffusion of Innovation.From the gradual weakening of the role of researchers to the gradual enhancement of the role of enterprises, there will be a weak stage.

The following part of this paper will introduce the evaluation system of scientific research results transformation maturity and BP neural network model to study the maturity of the transformation of scientific research achievements in the power industry. 


\section{Evaluation System and Bp Neural Network Model}

\subsection{Introduction of Scientific Research Results Transformation Maturity Evaluation System}

After achievement of scientific research can talk about achievement of scientific research to transform maturity. The factors influencing the transformation process from scientific research achievements are introduced above. The level of maturity of scientific research achievements transformation also needs to have a more perfect evaluation system.

The evaluation of the scientific and technological achievements of China Southern Power Grid is ultimately to improve the efficiency of the transformation of scientific and technological achievements. Therefore, this paper designs the evaluation index system of scientific and technological achievements according to the SMART criterion and the qualitative and quantitative principles. The process of transforming scientific and technological achievements is a very complicated social, economic and technological activity. On the basis of consulting relevant literature and materials and soliciting opinions from relevant experts, the process of transforming scientific and technological achievements as figure.1 is simplified into: technology source $\rightarrow$ scientific and technological achievements transformation activities $\rightarrow$ The external impact of the transformation of scientific and technological achievements (market) $\rightarrow$ the purpose of scientific and technological achievements transformation (effectiveness) four steps. At the same time, in order to ensure the systematic and comprehensive nature of the scientific and technological achievements evaluation index system, this paper is divided into three levels: target layer, criterion layer and indicator layer to construct the index system. Thus, an index system model with four steps as the vertical dimension and three levels as the horizontal dimension is formed.

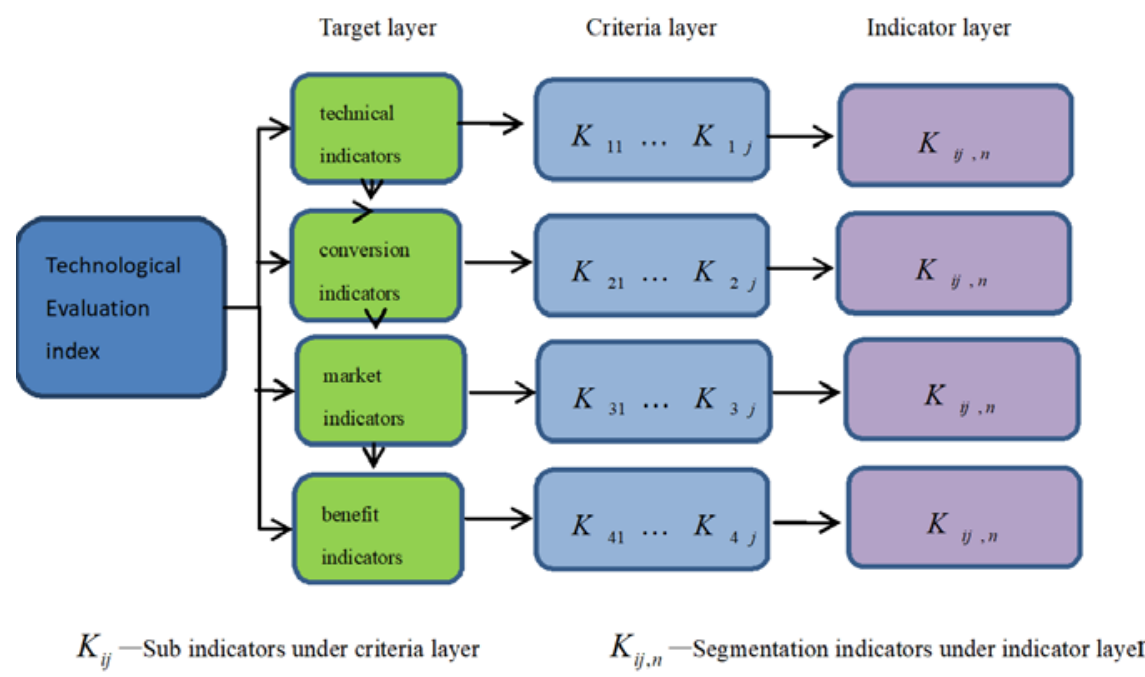

Fig.1 Technological Evaluation Index

Determination of evaluation indicators: According to the above-mentioned scientific and technological achievements evaluation index system model, starting from the four steps of the process of scientific and technological achievements transformation, the source of technology, the transformation of scientific and technological achievements, the external influence of scientific and technological achievements and The purpose of scientific and technological achievements transformation is the target level indicator. With reference to relevant references, the set of evaluation indicators is initially determined. According to the index system model of index scientific and technological achievements evaluation index, the whole set of indicator system consists of three layers: target layer, criterion layer and indicator layer. as shown in picture 2. The target layer includes five indicators: technical indicators, conversion indicators, market indicators, benefit indicators, and scientific indicators. The criteria layer includes 10 factors of evaluation of technical factors, technical risks, transformation organizations, transformation conditions, conversion risks, market factors, market risks, economic benefits, social benefits, and scientific factors. The indicator layer contains a total of 64 specific evaluation indicators. 


\subsection{Bp Neural Network Model}

BP (Back Propagation) neural network is one of the most widely used neural network models.Its structure includes input layer, hidden layer, and output layer.BP is a process in which the forward propagation of neurons and the simultaneous back propagation of errors occur.In other words, when there is deviation in the propagation process, the threshold and weight will be adjusted automatically, which is carried out from the output layer to the input direction. Through repeated learning, we finally get the threshold and weight of the minimum error compared with the expected value.

The comprehensive evaluation method based on BP artificial neural network has the advantages of fast calculation speed, high problem solving efficiency, strong self-learning ability and wide adaptability. It simulates the process of comprehensive evaluation by evaluation experts, so it is in the field of scientific and technological project evaluation. It has broad application prospects and can be widely used in the evaluation of various scientific and technological projects through the training of large sample data.

The BP neural network is a nonlinear algorithm with strong mapping ability. However, the BP neural network also has certain shortcomings in the running calculation. Because it is easy to fall into the local minimum, not the optimal solution of the whole nonlinear. In addition, the neural network is a calculation method in which the gradient is gradually reduced, so that the gradient disappears easily.

\subsubsection{Forward Transfer Process of Bp Neural Network}

Now set the weight $w_{i, j}$ between the node $i$ and the node $j$, the threshold of the node $j$ is $b_{j}$, the output value of each node is $x_{j}$. The output value of each node is realized according to the output value of all nodes in the upper layer, the weight of all nodes of the current node and the upper layer, the threshold of the current node, and the activation function.

$$
\begin{array}{r}
S_{j}=\sum_{i=0}^{m-1} w_{i j} x_{i}+b_{j} \\
x_{j}=f\left(S_{j}\right)
\end{array}
$$

Among them, $f$ is the activation function, generally, a sigmoid function or a linear function is selected. The input layer node has no threshold.

\subsubsection{Back Propagation}

In the BP neural network, the error signal back-transfer sub-process is more complicated. Suppose all the results of the output layer are $d_{j}$, The error function is as follows

$$
E(w, b)=\frac{1}{2} \sum_{j=0}^{n-1}\left(d_{j}-y_{j}\right)^{2}
$$

The BP neural network continually modifies the weights and thresholds so that the minimum error is kept close to $\sigma$. When the error is infinitely small and close to the expected value, the experiment ends.

\section{Results}

The article has already introduced the evaluation system for the value of scientific and technological achievements. There are 5 first-level indicators, 10 second-level indicators and 64 third-level indicators in the indicator system.Considering the process of transforming scientific and technological achievements, any sub-indicator plays an equal role in the weight of the overall indicator. By using the BP neural network, the weights obtained by considering the index layer and 
the weights obtained by taking the criterion layer and the index layer as variables are as shown in Table.1.

Table.1 the Weight of the Target

\begin{tabular}{|l|l|l|l|l|l|}
\hline Target layer & $\mathrm{K}_{1}$ & $\mathrm{~K}_{2}$ & $\mathrm{~K}_{3}$ & $\mathrm{~K}_{4}$ & $\mathrm{~K}_{5}$ \\
\hline Weight 1 & 0.2776 & 0.1881 & 0.1336 & 0.2388 & 0.1619 \\
\hline Weight 2 & 0.2799 & 0.1868 & 0.1302 & 0.2433 & 0.1598 \\
\hline
\end{tabular}

Both the criteria and indicator layers are weighted as variables to be closer to the actual value. The sum of ownership weights is equal to 1.It can be seen from the table that technical indicator has the highest weight and the market indicator has the lowest weight. From the analysis of the whole process to assess the relationship between the five indicators, it can be concluded that the source value of the technology (outcome) in the target layer is high, the value of the transformation activity of scientific and technological achievements is general, and the value of external factors (market) in the transformation of scientific and technological achievements is "Generally, the value of scientific research results transformation (benefit) is higher. It is found from the research results that the total value of scientific research results in the power industry is relatively high, but the stage from scientific research results to transformation is stuck, not smooth, resulting in low conversion rate of scientific research results.

\section{Conclusions}

This paper uses the BP neural network method, which shows that the transformation of scientific research results in the power industry is low. In view of the current low conversion rate of scientific research achievements in the power industry, there should be more economic and policy support to promote the development of scientific research results and create a better environment for innovation. Improve the transformation system of scientific and technological achievements. Promote talent development and team building. Increase investment in scientific research achievements. The assessment of technology needs to consider the many factors that extend in the development of economic production. This paper only analyzes the basic factors. More research is needed to include more influencing factors as indicators of evaluation.

\section{Acknowledgment}

Science and technology project of China Southern Power Grid Co., Ltd. (zbkjxm20180038).

\section{References}

[1] Zhao Yulin. Economic Analysis of the Transformation of Scientific and Technological Achievements [M]. Beijing: Enterprise Management Press, 2009.

[2] Tao Wei, research project selection evaluation based on credibility and decision threshold, value engineering,

[3] Gao Xinhua, Lu Yi, Current Status and Development of Research Values of China's Electric Power Research Institutes, Technology and Industry, 2013, 7

[4] Li Xiuquan, The Concept and Measurement Method of Transformation of Applied Science and Technology Achievements, Science and Technology Management Research, 2015.23

[5] Gao Xizhen, Liu Chaochao, "Study on Performance Evaluation Index System of Science and Technology Achievement Transformation Project Based on Government Perspective” Science and Technology Progress and Countermeasures. 2014.06

[6] He Defang. Analysis and thinking on some basic concepts of scientific and technological achievements and transformation of scientific and technological achievements [J]. National Soft Science, 2011 (11): 1-7

[7] E.M. Rogers.Diffusion of innovations.(fifth ed.), Free Press, New York (2003) 